\title{
Cerebrocostomandibular syndrome
}

INSERM

\section{Source}

INSERM. (1999). Orphanet: an online rare disease and orphan drug data base.

Cerebrocostomandibular syndrome. ORPHA:1393

Cerebro-costo-mandibular syndrome (CCMS) is characterized at birth by posterior rib gaps and orofacial anomalies reminiscent of Pierre Robin syndrome (see this term) that include palatal defects (short hard palate, absent soft palate, absent uvula), micrognathia and glossoptosis. 